\title{
Teaching medicine and biology through systems biology
}

\author{
H.V. Westerhoff \\ Synthetic Systems Biology and Nuclear Organization, University of Amsterdam \\ Molecular Cell Physiology, Vrije Universiteit Amsterdam \\ Systems Biology, The University of Manchester \\ Infrastructure Systems Biology Europe. NL \\ e-mail: h.v.westerhoff@uva.nl
}

Key words: hands-on modelling, understanding biological complexity, training life scientists

For long, the teaching of the Life Sciences focused on memorization of the metabolites, enzymes and pathways that were known. This gradually led to biochemistry and cell biology text books becoming extensive libraries in excess of half a million of words and to databases with the implementation of genomics. Some courses still require the students to learn these words by heart, and in the right sequence.

This practice is rapidly becoming obsolete, first by the number of facts growing exponentially whereas student brains do not, and second by Wikipedia taking over: students look up the components of biochemistry on their mobile phones faster than the Professor can pronounce them. Should we then refrain from teaching biology altogether? Some may say so, as they see Biology as just a large set of facts; a can of worms; a cacophony of special cases.

We take a rather diametrical position: we think that Biology and Medicine are complexity sciences, where most functions and malfunctions emerge in nonlinear interactions and are thereby hard to understand. And we hold that it is precisely this emergence that students should learn to understand. Yes, it is fine for them to look up the facts in proper databases: We move towards a teaching without facts but full of network mechanisms.

Here we shall demonstrate this teaching mode in hands-on training courses in which students train themselves to recognize and solve some of the paradoxes that abound in biology and medicine. They will be armed with blue-print computational models that they can then populate with facts looked up through Wikepedia. They will then be instructed how by running these models they can discover non-intuitive behaviour. And they will then interrogate the models further and resolve the paradoxes. This will make them realize why the MAPkinase route should better not be a MAPkinase route, why the most obvious may not be the best drug target, and why GWASH fails to elucidate the key component of most diseases. Networking enables functions that are otherwise thermodynamically impossible, such as the synthesis of ATP, proteins and DNA. We shall here highlight a lesser known function of networking, i.e. diversification. Network diversification followed by selection, sprouted the tree of Life, but that very tree hides a forest of diversity. Early Life on this planet may have benefitted from diversification of the redox network around acetogenesis. Flux Balance Analysis (FBA) of the genome-wide metabolic network of Cl. Ljungdahlii reveals carbon fixation at various ATP/acetate stoicheiometries. This may have enabled early organisms to survive the erratic environmental conditions by shifting gears. The flexible ATP yield enabled by the Warburg effect may help do so for tumor cells.

Our organs are subject to a drizzle of somatic mutations, which leads to cell diversification with age. We shall review an FBA methodology that simulates this and then demonstrate that this may initially enhance the metabolic versatility of organs such as T-cells and liver, in the absence of adaptation. More somatic mutations would cause loss of function and ageing. And, subpopulations of asocial cells would develop into tumors with Warburg and the new WarburQ (i.e. glutamine dependent) phenotypes.

Likewise, noise such as required by the third law of thermodynamics should diversify cell populations. FISH and deep sequencing experiments show even stronger noise than this, which should thereby be subject to regulation, e.g. through transcription bursting. We shall show that even though such noise varies with time, it may be selectable and may lead to drug resistance of tumor cell populations, either because of nonlinearities or because of the 'Waddington' genetic landscape, a remnant of developmental biology. 